\title{
Reactor anaerobio de flujo horizontal con medio de soporte de polietilentereftalato
}

\section{(Anaerobic horizontal flow reactor with polyethylene terephthalate as support material)}

\author{
Marcelo Muñoz ${ }^{1}$, Valeria Fuentes ${ }^{1}$, María Belén Aldás ${ }^{1}$
}

\begin{abstract}
Resumen:
Para eliminar la carga orgánica de aguas residuales de la industria lechera, se instaló un reactor anaerobio piloto, el cual usa un inóculo bacteriano aclimatado con anterioridad al sustrato. El reactor fue instalado horizontalmente y llenado con pedazos de polietilentereftalato (PET) de botellas de plástico. El reactor operó a temperatura ambiente, durante 100 días, en tres fases: 1) el reactor se estabilizó con una carga orgánica volumétrica de 0.013 a 0.500 $\mathrm{kg} / \mathrm{dí}^{\mathrm{a}} \mathrm{m}^{3}$; 2) el tiempo de retención hidráulico fue de 1 día y la carga orgánica volumétrica de 3 $\mathrm{kg} / \mathrm{día}^{3} \mathrm{~m}^{3}$; 3) la carga orgánica volumétrica se incrementó de 4 a $6.6 \mathrm{~kg} / \mathrm{día}^{\mathrm{m}} \mathrm{m}^{3}$ y el tiempo de retención hidráulico fue de 1 día. Se obtuvieron eficiencias de remoción de materia orgánica del $85 \%$, de las cuales aproximadamente el $75 \%$ se logró en la segunda y tercera fase, respectivamente. El valor de $Y$ fue de 0.15 , que indica que $0.15 \mathrm{~kg}$ de biomasa fueron generados por $\mathrm{kg}$ de DQO suministrado al reactor. Por último, la biomasa generada en el interior del reactor se analizó, obteniéndose un valor de $18868 \mathrm{mg} / \mathrm{L}$, que es un valor más alto que el de los sistemas convencionales.
\end{abstract}

Palabras clave: digestión anaerobia; reactor de flujo horizontal; biopelícula; agua residual; industria láctea.

\begin{abstract}
:
A pilot anaerobic reactor was installed to remove the organic load of wastewater from dairy industry. It uses a bacterial inoculum previously acclimated to the substrate. It was disposed horizontally and filled with pieces of polyethylene terephthalate (PET), from plastic bottles. The reactor was operated at room temperature, during 100 days, in three phases: 1) the reactor was stabilized with volumetric organic load from 0.013 to $0.500 \mathrm{~kg} /$ day. $\left.\mathrm{m}^{3} ; 2\right)$ the hydraulic retention time was of 1 day and the volumetric organic load of $3 \mathrm{~kg} / \mathrm{day}^{\mathrm{m}} \mathrm{m}^{3}$; 3 ) the volumetric organic load was incremented from 4 to $6.6 \mathrm{~kg} /$ day. $\mathrm{m}^{3}$ and the hydraulic retention time was 1 day. Organic material removal efficiencies was of $85 \%$, and approximately $75 \%$ were obtained in the second and third phase, respectively. The $Y$ value was 0.15 , indicating that $0.15 \mathrm{~kg}$ of biomass were generated by $\mathrm{kg}$ of QDO supplied to the reactor. Finally, the biomass generated inside the reactor was analyzed, obtaining a value of $18868 \mathrm{mg} / \mathrm{L}$, which is a higher value than those of conventional systems.
\end{abstract}

Keywords: anaerobic digestion; horizontal flow reactor; biofilm; wastewater; dairy industry.

\footnotetext{
${ }^{1}$ Escuela Politécnica Nacional, Quito - Ecuador (maria.aldas@epn.edu.ec)
} 


\section{Introducción}

La industria láctea genera gran cantidad de aguas residuales, las cuales provienen principalmente de las operaciones de lavado y limpieza dentro del proceso de producción. Varios autores indican que, por ejemplo en Europa, se ha verificado una producción diaria de $500 \mathrm{~m}^{3}$ para una industria láctea de tamaño mediano (Demirel, Yenigun y Onay, 2005). En India se ha estimado que la producción de agua residual es de una a tres veces el volumen de leche procesada (Tikariha y Sahu, 2014). Se estima que cerca del $2 \%$ de la leche procesada se vierte como residuo y que por litro de leche procesada se generan de 0.2 a 10 litros de agua residual, con un promedio de 2.5 litros (Kushwaha, Srivastava y Mall, 2011).

Las aguas residuales de la industria láctea se caracterizan por altas cargas orgánicas y valores de $\mathrm{pH}$ superiores a los rangos aceptables para vertimiento (Arango y Sanches, 2009). Poseen contenidos elevados de grasas y aceites, lactosa, proteínas solubles, sólidos suspendidos y sales minerales, y una elevada temperatura $\left(30-40^{\circ} \mathrm{C}\right.$ ) (Karadag, Köroğlu, Ozkaya y Cakmakci, 2015). Se han reportado variaciones en los valores de DQO que van desde 80 a $95000 \mathrm{mg} / \mathrm{L}$ y DBO de 40 a $48000 \mathrm{mg} / \mathrm{L}$. Se han observado valores de $\mathrm{pH}$ de 4.7 a 11, mientras que para los sólidos suspendidos se han reportado concentraciones de 0.024 a $4.5 \mathrm{~g} / \mathrm{l}$ (Kushwaha et al, 2011). Otros componentes importantes de las aguas residuales son los sanitizantes y detergentes, sustancias que por su carácter surfactante permiten la disolución de grasas y aceites en el agua residual facilitando la formación de biopelículas microbianas y flóculos de mayor tamaño y fácil sedimentación en procesos de biofiltración (Prócel, Posligua y Banchón, 2016).

Al llegar al cuerpo receptor, estas aguas residuales se descomponen rápidamente y consumen el oxígeno disuelto, lo que puede llevar a condiciones anaeróbicas, convirtiendo al cuerpo de agua en un lugar apto para el crecimiento de vectores de varias enfermedades, incluso se ha reportado que en altas cantidades estas aguas pueden ser tóxicas para ciertas especies de peces y algas (Kolhe, Ingale y Bhole, 2009).

Es prioritario entonces, aplicar un tratamiento a los efluentes finales de esta industria y varios han sido los procesos que se han desarrollado para el efecto, entre ellos aparecen los tratamientos físico químicos cuyos resultados reportados son variables, desde un 40\% de remoción de la materia orgánica en procesos de electrocoagulación-decantación con sales inorgánicas (TorresSánchez, Lopez-Cervera, de la Rosa, Maldonado-Vega, Maldonado-Santoyo y PeraltaHernandez, 2014) hasta un $87 \%$ en proceso de electrocoagulación aplicados a aguas residuales de industria de yogur (López y Harnisth, 2016). Estos tratamientos resultan costosos, por tanto es extensivo el uso de los tratamientos biológicos, dentro de los cuales se pueden mencionar lagunas, sistemas de lodos activados y tratamientos anaerobios, siendo uno de los métodos más utilizados el tratamiento anaerobio de biopelícula, para remover la materia orgánica contenida en este tipo de aguas (Demirel et al, 2005). 
Un reactor anaerobio es un equipo diseñado para que en su interior se lleve a cabo un proceso bacteriano sin la utilización de oxígeno, con algunas ventajas frente a los aerobios: baja generación de biomasa, bajos requerimientos nutricionales, producción de metano y no requerimiento de oxígeno; las características de este tipo de reactor lo hacen factible para el tratamiento del efluente de la industria láctea (Arango y Garcés, 2007).

Estos reactores suelen emplearse con un medio de soporte interno como: piedras, material plástico, mazorcas, cáscara de coco, entre otros, en cuya superficie la biomasa forma una película denominada biopelícula. La selección de un medio de soporte con características físicas y químicas adecuadas influye sobre la adherencia de la biopelícula; éstas son porosidad, estabilidad, superficie específica, entre otras (Solano y Rangel, 2006). El empleo de estos sistemas en la depuración de aguas residuales de la industria láctea ha presentado resultados que varían desde valores de $66 \%$ a $98.8 \%$ en la remoción de la DQO de aguas residuales tanto reales como sintéticas, con medios de soporte entre los que destacan cerámica, plásticos y arcillas (Karadag et al, 2014).

Por lo anterior, esta investigación pretende evaluar el comportamiento de un reactor anaerobio horizontal con material de soporte de polietilentereftalato (PET), para la eliminación de la carga orgánica del efluente de la industria láctea, con una perspectiva innovadora referente a la eficiencia de remoción de materia orgánica y concentración de biomasa en este tipo de reactor.

\section{Metodología}

Se utilizó un reactor horizontal y circular, de $50 \mathrm{~mm}$ de diámetro, elaborado en policloruro de vinilo (PVC), con material de relleno para tratar el efluente procedente de una industria de productos lácteos. El inóculo utilizado, proveniente de un río, fue un lodo anaerobio aclimatado con agua residual doméstica.

El reactor operó a temperatura ambiente, de aproximadamente $15^{\circ} \mathrm{C}$, con un caudal medio de 4 L/día, y con una DQO entre 1000 a 6000 mg/L, con concentración media de 3000 mg/L. Se operó con $\mathrm{pH}$ del afluente cercano a 8 para garantizar el crecimiento de bacterias metanogénicas; para esto fue necesario alcalinizar el medio utilizando bicarbonato de sodio. Se tomó como base de cálculo para el diseño una carga orgánica de $9.6 \mathrm{~g} / \mathrm{L}$ día, dato referido en la caracterización de un reactor anaerobio de flujo ascendente (UASB por sus siglas en inglés) que a la tasa indicada presentó adecuada eficiencia en la eliminación de materia orgánica, evaluado a temperatura ambiente (Arango y Sanches, 2009). El reactor se operó en forma discontinua con recirculación del efluente.

El sistema a escala piloto, que se observa en la Figura 1, estuvo conformado por: el reactor anaerobio horizontal (1), construido en tubería PVC, relleno con el material de soporte (7); el ingreso del afluente a tratar (2), salida del efluente tratado (5) y salida de gas (6). Contó con un 
sistema de recirculación formado por una bomba peristáltica (3) que recircula el fluido desde la salida del efluente (4) hasta el ingreso del afluente (2). Las mangueras de recirculación se cubrieron para evitar el ingreso de la luz y el crecimiento de algas. Para lograr la salida del metano el reactor se elevó $1 \mathrm{~cm}$ de la horizontal.

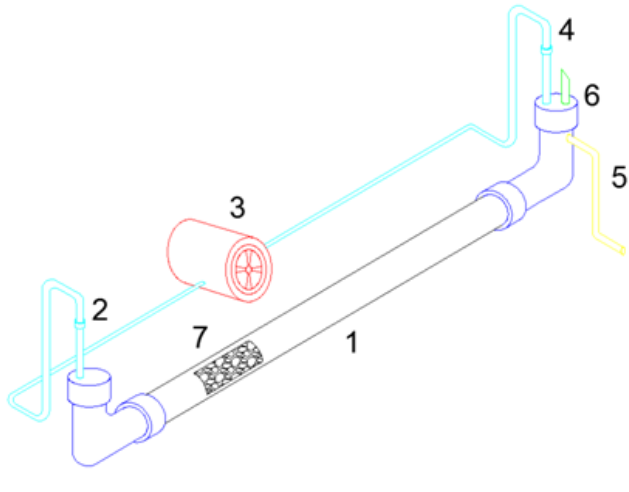

a)



b)

Figura 1. a) Esquema del reactor anaerobio horizontal b) reactor instalado

Como material de soporte para el crecimiento bacteriano se utilizó PET, plástico ampliamente utilizado en los envases de bebidas, en cortes de aproximadamente $1.5 \mathrm{~cm} \times 1.5 \mathrm{~cm}$, como se indica en la Figura 2.

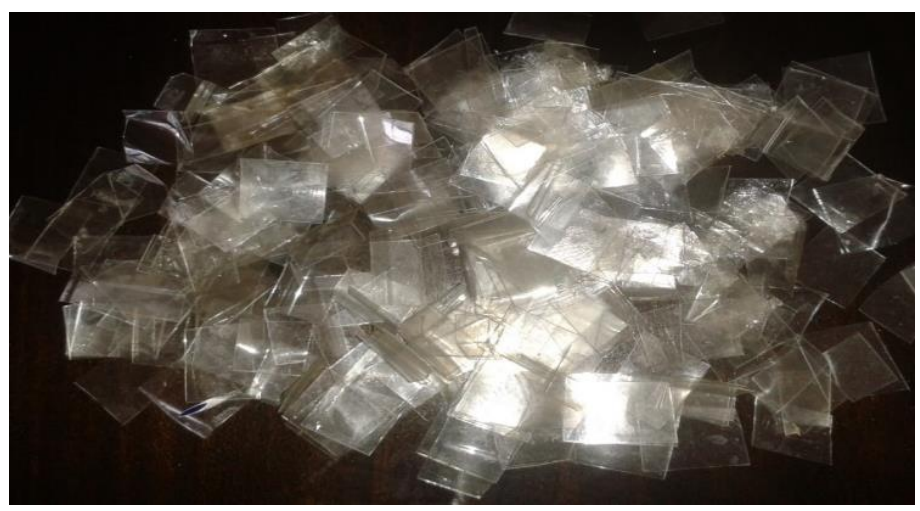

Figura 2. PET utilizado como relleno en el reactor anaerobio

En la Tabla 1 se encuentran registrados los principales parámetros del diseño del reactor anaerobio horizontal.

Se midieron los siguientes parámetros de control: $\mathrm{pH}$ usando un pHmetro/conductímetro Fisher Scientific accumet® AB15E; demanda química de oxígeno (DQO) por el método de digestión en reactor y uso del programa 430 DQO RB en espectrofotómetro HACH DR 2700; sólidos suspendidos totales (SST) y sólidos suspendidos volátiles (SSV) usando los protocolos estándar de la APHA-AWWA: procedimiento gravimétrico 103-105 ${ }^{\circ} \mathrm{C}, \mathrm{SM} 2540$ B para los sólidos totales y para sólidos volátiles procedimiento de ignición a $550^{\circ} \mathrm{C}, \mathrm{SM} 2540 \mathrm{E}$; tiempo de retención hidráulico (TRH); carga orgánica volumétrica (Lv); carga orgánica superficial (Ls) referida a la 
superficie del medio de soporte; relación Y (Biomasa - Carga Orgánica) y eficiencia de remoción de materia orgánica.

Tabla 1. Parámetros de diseño del reactor anaerobio

\begin{tabular}{|c|c|c|}
\hline Parámetro & Unidad & Valor \\
\hline Volumen total & $\mathrm{L}$ & 1.25 \\
\hline Volumen útil & $\mathrm{L}$ & 1.06 \\
\hline Diámetro del reactor & $\mathrm{mm}$ & 50 \\
\hline Largo del reactor & $\mathrm{m}$ & 0.64 \\
\hline Altura de la horizontal & $\mathrm{cm}$ & 1.0 \\
\hline Medio de soporte & - & Plástico PET \\
\hline Disposición del medio de soporte & - & Irregular $1.5 \mathrm{~cm} \times 1.5 \mathrm{~cm}$ \\
\hline
\end{tabular}

El arranque y operación del sistema duró cien días. El monitoreo del reactor se dividió en 3 etapas:

ETAPA I (etapa de arranque): se alimentó el reactor con agua residual de una industria láctea, con una concentración de $3000 \mathrm{mg} / \mathrm{L}$ de $\mathrm{DQO}$, con los valores de operación que se presentan en la Tabla 2.

Tabla 2. Parámetros de control etapa I

\begin{tabular}{|c|c|c|}
\hline $\begin{array}{c}\text { TRH } \\
\text { Días }\end{array}$ & $\begin{array}{c}\text { Carga volumétrica } \\
\text { afluente kg/día.m }\end{array}$ & $\begin{array}{c}\text { Carga orgánica } \\
\text { superficial kg/día.m }\end{array}$ \\
\hline 7 & \multicolumn{2}{|c|}{ Ingreso inóculo } \\
\hline 13 & 0.013 & 0.0000070 \\
\hline 11 & 0.064 & 0.0000338 \\
\hline 8 & 0.203 & 0.0001070 \\
\hline 3 & 0.660 & 0.0003472 \\
\hline 4 & 0.700 & 0.0003681 \\
\hline 6 & 0.500 & 0.0002629 \\
\hline
\end{tabular}

ETAPA II (operación): se monitoreó el sistema con una concentración constante de $3000 \mathrm{mg} / \mathrm{L}$ de DQO, un volumen constante del afluente y diferentes tiempos de retención hidráulicos, obteniendo los valores de la Tabla 3, hasta el día 65.

Tabla 3. Parámetros de control etapa II

\begin{tabular}{|c|c|c|}
\hline $\begin{array}{l}\text { TRH } \\
\text { días }\end{array}$ & $\begin{array}{l}\text { Carga volumétrica } \\
\text { afluente } \mathrm{kg} / \mathrm{dí}^{2} \cdot \mathrm{m}^{3}\end{array}$ & $\begin{array}{c}\text { Carga orgánica } \\
\text { superficial kg/día.m² }\end{array}$ \\
\hline 1.9 & 1.6 & 0.00084 \\
\hline 1.7 & 1.8 & 0.00095 \\
\hline 3.9 & 0.8 & 0.00043 \\
\hline 1.2 & 2.7 & 0.00144 \\
\hline 1.6 & 1.8 & 0.00097 \\
\hline 3 & 1.0 & 0.00052 \\
\hline
\end{tabular}

Luego del día 65 se operó con los valores representados en la Tabla 4, con un TRH constante de 1 día y $\mathrm{DQO}$ del afluente de $3000 \mathrm{mg} / \mathrm{L}$.

Tabla 4. Parámetros de control etapa II (TRH = 1 día)

\begin{tabular}{c|c|c}
\hline TRH & Carga volumétrica & Carga orgánica
\end{tabular}




\begin{tabular}{|c|c|c|}
\hline días & afluente $\mathrm{kg} / \mathrm{día} \cdot \mathrm{m}^{3}$ & superficial kg/día.m ${ }^{2}$ \\
\hline 1 & 3 & 0,0016 \\
\hline 1 & 3 & 0,0016 \\
\hline 1 & 3 & 0,0016 \\
\hline 1 & 3 & 0,0016 \\
\hline 4 & 0.75 & 0,0004 \\
\hline 1 & 3 & 0,0016 \\
\hline 1 & 3 & 0,0016 \\
\hline 1 & 3 & 0,0016 \\
\hline 3 & 1 & 0,0005 \\
\hline 1 & 3 & 0,0016 \\
\hline 1 & 3 & 0,0016 \\
\hline 1 & 3 & 0,0016 \\
\hline
\end{tabular}

ETAPA III (operación): se operó el sistema aumentando las concentraciones para verificar la máxima concentración de DQO que el reactor anaerobio horizontal podría tratar, a un volumen constante, con un $\mathrm{TRH}=1$ día. En la Tabla 5 se presentan estos valores.

Tabla 5. Parámetros de control etapa III

\begin{tabular}{|c|c|c|}
\hline $\begin{array}{c}\text { TRH } \\
\text { días }\end{array}$ & $\begin{array}{c}\text { Carga volumétrica } \\
\text { afluente } \mathbf{k g} / \text { día. }^{\mathbf{3}}\end{array}$ & $\begin{array}{c}\text { Carga orgánica } \\
\text { superficial kg/día.m2 }\end{array}$ \\
\hline 1 & 4.0 & 0.0021 \\
\hline 1 & 4.5 & 0.0024 \\
\hline 1 & 5.0 & 0.0026 \\
\hline 1 & 5.5 & 0.0029 \\
\hline 2 & 3.0 & 0.0016 \\
\hline 3 & 2.0 & 0.0011 \\
\hline 1 & 6.0 & 0.0032 \\
\hline 1 & 6.0 & 0.0032 \\
\hline 1 & 6.0 & 0.0032 \\
\hline 1 & 6.1 & 0.0032 \\
\hline 3 & 2.1 & 0.0011 \\
\hline 1 & 6.3 & 0.0033 \\
\hline 1 & 6.6 & 0.0035 \\
\hline
\end{tabular}

Terminada la etapa de operación se analizó la biopelícula que se formó en el reactor anaerobio horizontal, que fue desprendida del medio de soporte, y sometida a análisis de sólidos totales y sólidos totales volátiles.

\section{Resultados y discusión}

En la Figura 3, se presenta el comportamiento del reactor anaerobio horizontal en función de la carga orgánica volumétrica en las tres etapas de monitoreo; se puede observar cómo influye la cantidad de materia orgánica suministrada en determinado tiempo por unidad de volumen del reactor en el metabolismo celular, en este caso perjudicándolo y en consecuencia disminuyendo las eficiencias de remoción. 




Figura 3. Lv afluente - Eficiencia vs. Tiempo

Durante la ETAPA I se adaptó el sistema con aumentos en la carga orgánica volumétrica hasta 3 $\mathrm{kg} / \mathrm{día} \cdot \mathrm{m}^{3}$, alcanzando eficiencias de $94 \%$ (día 46), con Lv afluente $=0.7 \mathrm{~kg} / \mathrm{dí}^{2} \cdot \mathrm{m}^{3}$. En la ETAPA II se mantuvo una carga orgánica volumétrica de $3 \mathrm{~kg} / \mathrm{día} \cdot \mathrm{m}^{3}$, obteniéndose eficiencias entre el $75 \%$ y $85 \%$ en remoción de materia orgánica. Se obtuvo un $87 \%$ de eficiencia (día 81 ), con Lv afluente = $3 \mathrm{~kg} /$ día $\mathrm{m}^{3}$. En la ETAPA III se obtuvieon eficiencias máximas de un 77\% (día 93), con Lv afluente $=6 \mathrm{~kg} /$ día $\cdot \mathrm{m}^{3}$. Se obtuvieron eficiencias del 84\% (día 83) y 78\% (día 85) para cargas volumétricas de 4 y $5 \mathrm{~kg} / \mathrm{dí}^{2} \mathrm{~m}^{3}$, respectivamente. Se observa que a medida que se aumenta la carga volumétrica desde 6.2 a $6.6 \mathrm{~kg} /$ día $^{3} \mathrm{~m}^{3}$ en el afluente la eficiencia de remoción desciende hasta un 70\% (día 100). Esto debido a que con el incremento de carga orgánica suministrada, la biomasa presenta dificultad en consumir la materia orgánica, por lo que la eficiencia tiende a disminuir.

Por otro lado, en la Figura 4 se puede observar que el aumento de la carga orgánica con respecto al área superficial de la biopelícula, también genera condiciones que afectan negativamente el crecimiento celular; se acelera la fase acidogénica de la digestión anaerobia, lo que genera un desbalance en el metabolismo celular y por ende una caída de le eficiencia del reactor. A medida que la carga orgánica superficial aumenta, la eficiencia en el reactor anaerobio disminuye; las eficiencias son mayores cuando el reactor opera con cantidades menores a $0.0010 \mathrm{~kg}$ DQO/día.m². 




Figura 4. Ls - Eficiencia vs. Tiempo

Se observa que con concentraciones de DQO afluente por debajo de $6000 \mathrm{mg} / \mathrm{L}$, se obtienen concentraciones medias de DQO efluente inferiores de $1400 \mathrm{mg} / \mathrm{L}$, mientras que al operar con $6600 \mathrm{mg} / \mathrm{L}$ se obtienen concentraciones medias del DQO del efluente de alrededor de $2000 \mathrm{mg} / \mathrm{L}$; esto se aprecia en la Figura 5. La máxima eliminación de materia orgánica fue del 87\%, 84\%, 78\% y $77 \%$ para cargas orgánicas de 3, 4,5 y $6 \mathrm{~kg} / \mathrm{m}^{3}$ por día respectivamente, con un tiempo de retención hidráulico de un día luego de 81, 83, 85 y 94 días de operación, respectivamente.



Figura 5. Relación DQO afluente y efluente - eficiencia vs. Tiempo 
El reactor anaerobio dejó de operar al presentar un descenso de la remoción de materia orgánica a medida que la concentración de DQO aumentaba en el afluente. En las dos últimas cargas se verificó que el crecimiento de biomasa provocó la saturación en el sistema presentando inconvenientes como pérdida de biomasa en el efluente del reactor y el taponamiento del mismo.

Al respecto, Arango y Sánchez (2009) mencionan en su investigación que al aumentar la carga orgánica en los reactores, el crecimiento de las bacterias fue favorecido; sin embargo la tasa de crecimiento de los microorganismos acidogénicos es cinco veces superior a la de los microorganismos metanogénicos, ocasionando mayor generación de productos intermedios resultantes de la acidificación, que la biomasa metanogénica no es capaz de metabolizar totalmente, por lo cual se produce una disminución en el porcentaje de eliminación de materia orgánica.

Porcentajes de remoción de materia orgánica similares a los obtenidos han sido reportados en investigaciones realizadas en un reactor UASB seguido de un sistema de lodos activados para el tratamiento de aguas de descarga de una industria láctea, en los que el reactor UASB trabajó con un tiempo de retención hidráulico de 24 horas y cargas orgánicas volumétricas en rango de 1.9 a $4.4 \mathrm{~kg} / \mathrm{m}^{3}$.día, alcanzándose remociones de DQO y DBO del 69\% y 79\%, respectivamente (Tawfik, Sobhey y Badawy, 2008).

Otras investigaciones mostraron que un reactor UASB alcanzó sobre un 80\% - 90 \% de remoción de DQO y DBO bajo una carga orgánica volumétrica de $6 \mathrm{~kg} / \mathrm{m}^{3}$.día, con un tiempo de retención de 20 horas (Ince, 1998).

En estudios similares utilizando el efluente de la industria láctea, Campos y Parra (2014) obtuvieron eficiencias cercanas al $80 \%$ con cargas de $3 \mathrm{~kg}$ DQO/L.día, a condiciones de temperatura ambiente $\left(18-20^{\circ} \mathrm{C}\right)$.

En el trabajo realizado por Rodríguez, Pérez, Rodríguez de la Garza y Garza (2005), el cual utiliza aguas residuales de lácteos a una temperatura de 25 a $60^{\circ} \mathrm{C}$, se menciona que por encima de 6 g/L.día la eficiencia de remoción del reactor UASB disminuía.

Se ha probado también la eficiencia de filtros anaerobios con medios de soporte porosos y no porosos, donde se ha encontrado que los reactores con medios no porosos muestran inestabilidad a cargas orgánicas volumétricas mayores a $4 \mathrm{~kg} / \mathrm{m}^{3}$.día, lo que indica que el reactor horizontal ensayado presenta mejor desempeño para cargas de hasta $6.6 \mathrm{~kg} / \mathrm{m}^{3}$.día (Anderson, Kasapgil e Ince, 1994).

Si se considera el área del soporte PET $2.016 \mathrm{~m}^{2}$ y el volumen útil del reactor $0.00106 \mathrm{~m}^{3}$, el reactor anaerobio horizontal presentó una superficie específica $1902 \mathrm{~m}^{2} / \mathrm{m}^{3}$. 
El reactor anaerobio horizontal tiene una relación $Y=0.15$, es decir, por cada $\mathrm{kg}$ de DQO alimentado se desarrolló $0.15 \mathrm{~kg}$ de biomasa expresada como SSV.

En un estudio realizado en un reactor anaerobio de lecho móvil, alimentado con aguas residuales de quesería, bajo condiciones mesofílicas, se encontró que la relación Y fue de 0.0794 (Wang, Chandrasekhara Rao, Qu y Moletta, 2009) lo que indica que el reactor ensayado produce mayor cantidad de biomasa por $\mathrm{kg}$ de DQO.

Del análisis de SST y SSV se obtuvo los resultados mostrados en la Tabla 6.

Tabla 6. Resultados análisis de biomasa del reactor anaerobio

\begin{tabular}{|c|c|c|}
\hline Parámetro & Unidad & Valor \\
\hline Sólidos totales, SST & $\mathrm{g}$ & 32.0 \\
\hline Sólidos volátiles totales, SSV & $\mathrm{g}$ & 20.0 \\
\hline
\end{tabular}

Con los datos anteriores, al considerar que el volumen útil del reactor es de $1.06 \mathrm{~L}$, se determinó que este tiene una concentración de biomasa de $18868 \mathrm{mg} / \mathrm{L}$, que es un valor considerablemente elevado, comparado con los obtenidos en sistemas de tratamiento convencionales.

\section{Conclusiones y Recomendaciones}

Es factible la operación de un reactor anaerobio horizontal con material de soporte PET, para el tratamiento de las aguas residuales de la industria láctea en condiciones de temperatura ambiente; los microorganismos presentan una adaptación a condiciones psicrofílicas (menor a 20 ${ }^{\circ} \mathrm{C}$ ), con lo cual se obtiene una eficiente actividad microbiana.

El acondicionamiento del afluente con bicarbonato de sodio permitió mantener el afluente en el tiempo de tratamiento, en un ambiente alcalino con un rango de $\mathrm{pH}$ (7.5 - 8); esto favoreció la digestión anaerobia.

El reactor anaerobio de flujo horizontal operó en condiciones estables para el tratamiento de aguas residuales de la industria láctea, con carga orgánica desde 0.013 hasta $6.2 \mathrm{~kg} / \mathrm{m}^{3}$.día, ya que a cargas superiores se observó una disminución en la eficiencia de remoción de materia orgánica y una sobrecarga en el sistema.

La adaptación del sistema de recirculación del afluente favoreció la remoción de materia orgánica debido al mayor contacto del afluente con la biopelícula; además se verificó la formación de la biopelícula en el material de soporte PET. El material de soporte utilizado es un material de desecho, bajo costo, de fácil consecución y presenta estabilidad química. 


\section{Bibliografía}

Anderson, G.K., Kasapgil, B., Ince, O. (1994). Comparison of porous and non-porous media in upflow anaerobic filters when treating dairy wastewater. Water Research. (28) 7, 1619-1624. doi:10.1016/0043-1354(94)90229-1

Arango O., Sanches, L. (2009). Tratamiento de aguas residuales de la industria láctea en sistemas anaerobios tipo UASB. Facultad de Ciencias Agropecuarias (9) 2, 24-31.

Arango A., Garcés, F. (2007). Tratamiento de aguas residuales de la industria láctea. Producción más limpia, (2) 2, 23-30.

Campos, R. G., Parra, R. A. (2015). Evaluación del comportamiento de un reactor UASB con diferentes cargas orgánicas provenientes de lactosuero. Producción + Limpia, 9(1), 23-30.

Demirel, B., Yenigun, O., Onay, T. T. (2005). Anaerobic treatment of dairy wastewaters: a review. Process Biochemistry, 40(8), 2583-2595. doi:10.1016/j.procbio.2004.12.015

Ince, O. (1998). Potential energy production from anaerobic digestion of dairy wastewater. Journal of Environmental Science and Health, Part A: Toxic/Hazardous Substances and Environmental Engineering. (33) 6, 1219-1228. doi:10.1080/10934529809376784

Karadag, D., Köroğlu, O. E., Ozkaya, B., Cakmakci, M. (2015). A review on anaerobic biofilm reactors for the treatment of dairy industry wastewater. Process Biochemistry, 50(2), 262271. doi:10.1016/j.procbio.2014.11.005

Kolhe, A. S., Ingale, S. R., Bhole, R. V. (2009). Effluent of dairy technology. Shodh Samiksha aur Mulyankan (International Research Journal), 2, 459-461.

Kushwaha, J. P., Srivastava, V. C., Mall, I. D. (2011). An overview of various technologies for the treatment of dairy wastewaters. Critical reviews in food science and nutrition, 51(5), 442-452. DOI:10.1080/10408391003663879

López, P., Harnisth, A. (2016). Electrocoagulación de aguas residuales de la industria láctea. Enfoque UTE, 7(1), 13-21.

Prócel, D., Posligua, P., Banchón, C. (2016). Biodegradación de contaminantes orgánicos de la industria láctea. Enfoque UTE, 7(1), 22-32. 
Rodríguez, J., Pérez, D., de la Garza, J. A. R., Garza, Y. (2005). Evaluación cinética de las aguas residuales de la industria láctea en sistemas batch y un reactor UASB. XI Congreso de Biotecnología y Bioingeniería Sociedad Mexicana de Biotecnología y Bioingeniería, Yucatán, México.

Solano J., Rangel, M. (2006). Evaluación operacional de un sistema a escala laboratorio de biopelícula anaerobia soportada para el tratamiento de aguas residuales domésticas (tesis de pregrado). Universidad Industrial de Santander, Bucaramanga, Colombia.

Tawfik, A., Sobhey, M., Badawy, M. (2008). Treatment of a combined dairy and domestic wastewater in an up-flow anaerobic sludge blanke (UASB) reactor followed by activated sludge (AS system). Desalination, (227) 1, 167-177. doi:10.1016/j.desal.2007.06.023

Tikariha, A., \& Sahu, O. (2014). Study of characteristics and treatments of dairy industry waste water. Journal of applied \& environmental microbiology,2(1), 16-22. DOI:10.12691/jaem-2-14

Torres-Sanchez, A. L., Lopez-Cervera, S. J., de la Rosa, C., Maldonado-Vega, M., MaldonadoSantoyo, M., Peralta-Hernandez, J. M. (2014). Electrocoagulation process coupled with advance oxidation techniques to treatment of dairy industry wastewater. International Journal of Electrochemical Science, 9, 6103-6112.

Wang, S., Chandrasekhara Rao, N., Qiu, R. Moletta, R. (2009). Performance and kinetic evaluation of anaerobic moving bed biofilm reactor for treating milk permeate from dairy industry. Bioresource Technology, (100) 23, 5641-5647. doi:10.1016/j.biortech.2009.06.028 